\title{
Chronic pain affected the way individuals viewed their bodies, their relationships with others, and their sense of time
}

Thomas SP.A phenomenologic study of chronic pain. West J Nurs Res 2000 Oct;22:683-99.

\section{QUESTION: What are the perceptions of adults living with chronic pain?}

Design

Eidetic (descriptive) phenomenology.

Setting
USA.

\section{Participants}

13 adults (age range $27-79$ y, $69 \%$ women, $92 \%$ white, $77 \%$ married) who were $>18$ years of age, willing to talk about their lived experiences, and with a duration of non-malignant chronic pain ranging from 7 months to 41 years, were recruited via a newspaper article and network sampling. Pain was most often located in the back, but also in the shoulder, arm, neck, hip, leg, jaw, and ear.

\section{Methods}

In individual 1-2 hour interviews, participants were asked to describe what it was like to live with chronic pain. The interviews were audiotaped and transcribed verbatim. The transcriptions were analysed independently by the researcher, and then read aloud and discussed by an interdisciplinary research group. Global themes were clustered to produce a thematic structure of the phenomenon.

\section{Main findings}

Findings fit into the context of the 3 existential grounds of body, other people, and time. Although the researchers also sought to place findings in the context of the world, the participants rarely commented on it, and seemed to have little awareness of a world external to their pain. Because of chronic pain, the participants were continually aware of having a body (in contrast to healthy individuals' relative lack of consciousness of physical self) that housed their pain and was the main focus of their existence. Participants saw the body as an obstacle rather than an enabler to action. Participants noted the incongruity between thoughts of productive activity and physical inability to enact their intentions. The pain often overtook the mind as well as the body, blocking all other sensation or thought. Participants also noted chronic pain as a physical disorder invisible to the people around them. Participants felt separated from other people because of their chronic pain, and hid their condition to avoid adverse reactions from others. They felt their pain isolated them, yet they were intimately connected to their internal "tormentor," "opponent," or "monster," their chronic pain. Contact with others occurred most often in the context of appointments with a physician, whom participants approached with both hope and mistrust. Few participants mentioned a support person with whom they could freely talk about their pain. The unit of time most important to the participants was the "moment," a concept that defined not only the present time but also a possible eternity of suffering. In the agony of the moment, they experienced a stopping of time as if the moment would never end. The pain also made participants more aware of their aging. After a cyclical process of seeking a cure, many participants came to see treatment as futile and talked about death as a source of final relief from their pain. Only 3 participants perceived positive aspects related to their chronic pain such as facilitating interaction with others and knowledge of self.

\section{Conclusions}

Participants with chronic pain experienced and perceived the world through the filter of their pain. Chronic pain affected the way they viewed their bodies, their relationships with others, and their sense of time. They had little perception of the world external to their pain.

\section{COMMENTARY}

In this phenomenological study, Thomas provides valuable insight into the lives of individuals experiencing nonmalignant chronic pain. 13 individuals, mostly white married women with varying duration of chronic pain, identified the essence of their pain experience as "unremitting torment, by a force or monster that cannot be tamed." Although the author sought variability in the sample to more clearly identify the phenomenon of pain, only one black woman and 4 men participated in the study. Additional work focusing on cultural, developmental, and gender based issues related to the experience and societal response to chronic pain would enhance understanding of these factors as salient considerations in pain assessment and effective management of patients' pain.

Through analysis of audiotaped interviews by an interdisciplinary team, various figural themes assumed prominence within the context of "body, other people, and time," a framework for the phenomenological approach derived from the Husserlian tradition used in this study. Participant data illustrate the consuming and controlling nature of chronic pain. Most participants denied the transformative nature of chronic pain despite earlier research supporting this experience as beneficial or strengthening to chronically ill individuals. Although actively involved in and ethically bound to deliver quality care to all people, nurses were not perceived by participants as supportive of their pain experience. Additional investigation needs to be completed to discover reasons for non-responsiveness of nursing staff to patient perspectives of pain because of its important influence on patients' defined quality of life. The power of chronic pain to negatively influence a person's definition of a good life demands that healthcare providers effectively assess patients' perceptions of pain and then collaborate with them to implement effective strategies to improve their lives.

Carolyn Spence Cagle, RNC, PhD Associate Professor, Harris School of Nursing College of Health and Human Sciences Texas Christian University Fort Worth, Texas, USA
USA.Fax +1865974

3569 . 